\title{
Financial Forecasting Using the Kolmogorov-Feller Equation
}

Jonathan Blackledge

Technological University Dublin, jonathan.blackledge@tudublin.ie

Marc Lamphiere

Technological University Dublin, marc.lamphiere@gmail.com

Kieran Murphy

TradersNow Limited, kieran@tradersnow.com

See next page for additional authors

Follow this and additional works at: https://arrow.tudublin.ie/engscheleart2

Part of the Electrical and Computer Engineering Commons

\section{Recommended Citation}

Blackledge, J., Lamphiere, M., Murphy, K., Overton. Financial Forecasting Using the Kolmogorov-Feller Equation. IAENG Transactions on Engineering Technologies Lecture Notes in Electrical Engineering, 229. Springer Science+Business Media, 2013. doi:10.1007/978-94-007-6190-2_50

This Article is brought to you for free and open access by the School of Electrical and Electronic Engineering at ARROW@TU Dublin. It has been accepted for inclusion in Articles by an authorized administrator of ARROW@TU Dublin. For more information, please contact arrow.admin@tudublin.ie, aisling.coyne@tudublin.ie, gerard.connolly@tudublin.ie.

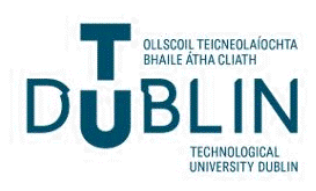


Authors

Jonathan Blackledge, Marc Lamphiere, Kieran Murphy, and Shaun Overton

This article is available at ARROW@TU Dublin: https://arrow.tudublin.ie/engscheleart2/75 


\title{
Chapter 1
}

\section{FINANCIAL FORECASTING USING THE KOLMOGOROV-FELLER EQUATION}

\author{
JONATHAN BLACKLEDGE
}

Dublin Institute of Technology, Kevin Street, Dublin 8, Ireland, jonathan.blackledge@dit.ie

\author{
MARC LAMPHIERE
}

Dublin Institute of Technology, Kevin Street, Dublin 8, Ireland, marclamphier@gmail.com

KIERAN MURPHY

TradersNow Limited, 15 Cabinteely Way, Cabinteely, Dublin 18, Ireland, kieran@tradersnow.com

\section{SHAUN OVERTON}

2665 Villa Creek Dr, Suite 125, Dalla, Texas 75234, USA, soverton@onestepremoved.com

Abstract An approach to analysing a financial time series using the KolmogorovFeller Equation is considered, in particular, the Generalised KolmogorovFeller Equation (GKFE), subject to variations in the Stochastic Volatility. Using the Mittag-Leffler memory function, we derive an expression for the Impulse Response Function associated with a short time window of data which is then used to derive an algorithm for computing a new index using a standard moving window process. It is shown that application of this index to financial time series, subject to a low volatility condition, correlates with the start, direction and end of a trend depending on the sampling rate of the time series and the look-back window or 'period' that is used. An example of this is provided in the paper using MetaTrader4.

Keywords: Generalised Kolmogorov-Feller Equation, Impulse Response Function, MetaTrader4, Mittag-Leffler Memory Function, Time Series Analysis, Trend Analysis, Stochastic Volatility. 


\section{Introduction}

This paper follows the work of Blackledge et al., 2012, extending the material to include an implementation of the results using MetaTrader 4. Beta tested MetaTrader4 functions are provided for those readers interested in implementing the approach considered, analysing other financial time series data and extending the algorithms further.

Price models involve the derivation and solution of a variety of stochastic differential and partial differential equations. Conider the 'standard model' for the price of a stock as a function of time $s(t)$ given by (e.g. Blackledge, 2010)

$$
\frac{d}{d t} s(t)=\mu s(t)+\sigma s(t) u(t)
$$

where $\mu$ is the 'Drift', $\sigma$ is the 'Volatility' and $u(t)$ is a stochastic function. This model is based on the idea that prices appear to be the previous price plus some random change and that these price changes are independent, i.e. asset price changes appear to be random and independent, prices being taken to follow some random walk-type behaviour. This is the basis for including a stochastic function $u(t)$. However the size of price movements also depends on the size of the price itself. The model is therefore revised to include this effect, the stochastic term $u(t)$ being replaced by $u(t) s(t)$ where $\sigma$ determines the degree of randomness taken to influence a price change. In general, $\mu$ and $\sigma$ vary with time, and, in the context of equation (1.1), $\sigma(t)$ is referred to as the 'Stochastic Volatility', e.g. Lamoureux and Lastrapes, 1993, Wiggins, 1987 and Melino and Turnbull, 1990. The drift function $\mu(t)$ tends to vary over longer periods of time reflecting the long term trends associated with a price index.

In principle, $u(t)$ could be any stochastic function with statistical behaviour conforming to a range of Probability Density Functions. A conventional model is to assume that the log price changes are Gaussian distributed so that $u(t)$ is taken to be a zero-mean Gaussian distributed function. If this function is taken to have a fixed standard deviation of 1 , then the volatility becomes a measure of the standard deviation, at least, for a (zero-mean) Gaussian model. The stock price model given by equation (1) then provides a method for estimating the volatility $\sigma$ in terms of a lower bound as discussed in the following section.

In this paper, we consider a solution to the Generalised KolomogorovFeller Equation to model the stochastic behaviour of a financial time series. By defining an Impulse Response Function which is based on a parameter associated with the Mittag-Leffler memory function used to construct the KFE, we consider an algorithm for analysing the trends of the time series. 


\section{Evaluation of the Stochastic Volatility}

Consider the rate equation

$$
f(t)=\mu+\sigma u(t)
$$

where

$$
f(t)=\frac{1}{s(t)} \frac{d}{d t} s(t)=\frac{d}{d t} \ln s(t)
$$

and $\mu$ and $\sigma$ are taken to be constant. We first obtain an estimate of the Drift by noting that, if the mean of $u(t)$ is approximately zero over $t \in[0, T]$, then

$$
\int_{0}^{T} f(t) d t=\int_{0}^{T} \mu d t+\sigma \int_{0}^{T} u(t) d t \sim \mu T
$$

so that

$$
\mu \sim \frac{1}{T} \int_{0}^{T} f(t) d t
$$

To obtain an estimate for the volatility, we now consider the case when the stochastic function $u(t)$ is a phase only function, i.e. given that

$$
\widetilde{u}(\omega)=\int_{-\infty}^{\infty} u(t) \exp (-i \omega t) d t
$$

where $\omega$ is the (angular) frequency, we consider

$$
\widetilde{u}(\omega)=A \exp [i \theta(\omega)]
$$

where the amplitude spectrum $A$ is taken to be a constant for all values of $\omega$. We also consider $u(t)$ to be a band-limited function $\omega \in[-\Omega / 2, \Omega / 2]$ with bandwidth $\Omega$ and a function of compact support $t \in[-T / 2, T / 2]$. Using Minkowski's identity for Euclidean norms,

$$
\|f(t)\|_{2} \leq\|\mu\|_{2}+\|\sigma u(t)\|_{2}
$$

where

$$
\|x(t)\|_{2}:=\left(\int|x(t)|^{2} d x\right)^{\frac{1}{2}}
$$

so that we can write

$$
\sigma\|u(t)\|_{2} \geq\|f(t)\|_{2}-\mu \sqrt{T}
$$


where $\mu$ is given by equation (1.2). Using Parseval's Theorem (Rayleigh's Energy Theorem), the condition expressed by equation (1.3) allows us to write

$$
\int_{-T / 2}^{T / 2}|u(t)|^{2} d t=\frac{1}{2 \pi} \int_{-\Omega / 2}^{\Omega / 2}|\widetilde{u}(\omega)|^{2} d \omega=\frac{\Omega A^{2}}{2 \pi}
$$

We can therefore consider the equation

$$
\sigma_{\min }=\frac{1}{A} \sqrt{\frac{2 \pi}{\Omega}}\left(\|f(t)\|_{2}-\mu \sqrt{T}\right)
$$

which yields an expression for the lower bound of the volatility.

\section{Numerical Computation of the Stochastic Volatility}

Consider a discrete signal denoted by the array $f_{n}, \quad n=1,2,3, \ldots, N$ where a uniform sampling interval of $\Delta t$ is assumed. In this case, the discrete version of equation (1.4) becomes

$$
\sigma_{\min }=\frac{1}{A} \sqrt{\frac{2 \pi}{\Omega}}\left(\sqrt{\Delta t}\left\|f_{n}\right\|_{2}-\mu \sqrt{T}\right)
$$

where we invoke the usual definition for a vector (Euclidean) norm, i.e.

$$
\left\|f_{n}\right\|_{2}:=\left(\sum_{n=1}^{N}\left|f_{n}\right|^{2}\right)^{\frac{1}{2}}, \quad \mu=\frac{\Delta t}{T} \sum_{n=1}^{N} f_{n}
$$

The sampling interval $\Delta t$ of $f_{n}$ is related to the sampling interval $\Delta \omega$ of the Discrete Fourier Transform of $f_{n}$ by the equation

$$
\Delta t \Delta \omega=\frac{2 \pi}{N}
$$

and since the bandwidth of the discrete spectrum of $f_{n}$ is $N \Delta \omega$ is is clear that $\Delta t=2 \pi / \Omega$. Thus, given that the support of the signal is $T=N \Delta t$, we note that

$$
T=\frac{2 \pi N}{\Omega}
$$

and therefore obtain

$$
\sigma_{\min }=\frac{2 \pi}{A \Omega}\left(\left\|f_{n}\right\|_{2}-\sqrt{N} \mu\right), \quad \mu=\frac{1}{N} \sum_{n=1}^{N} f_{n}
$$


The scaling constant $2 \pi /(A \Omega)$ can then be used to define a re-scaled Stochastic Volatility given by

$$
\hat{\sigma}:=\sigma_{\min } \frac{A \Omega}{2 \pi}
$$

thereby yielding the expression

$$
\hat{\sigma}=\left\|f_{n}\right\|_{2}-\sqrt{N} \mu
$$

Writing this result explicitly in terms of the price value $s_{n}$ we obtain the equation

$$
\hat{\sigma}=\left(\sum_{n=1}^{N-1}\left|\ln \left(\frac{s_{n+1}}{s_{n}}\right)\right|^{2}\right)^{\frac{1}{2}}-\frac{1}{\sqrt{N-1}} \sum_{n=1}^{N-1} \ln \left(\frac{s_{n+1}}{s_{n}}\right)
$$

To compute the 'Stochastic Volatility' $\sigma_{m}, N$ is taken to determine the size of the data sampling window or 'look-back' window which is moved along the time series one element at a time so that we can write

$$
\hat{\sigma}_{m}=\left(\sum_{n=1}^{N-1}\left|\ln \left(\frac{s_{m+n+1}}{s_{m+n}}\right)\right|^{2}\right)^{\frac{1}{2}}-\frac{1}{\sqrt{N-1}} \sum_{n=1}^{N-1} \ln \left(\frac{s_{m+n+1}}{s_{m+n}}\right)
$$

Equation (1.5) may be compared with other estimates for the Stochastic Volatility such as the Maximum Likelihood (ML) estimate given by, Stein and Stein, 1991

$$
\hat{\sigma}_{\mathrm{ML}}^{2}=\frac{1}{N-1} \sum_{n=1}^{N-1}\left[\ln \left(\frac{s_{n+1}}{s_{n}}\right)\right]^{2}-\frac{1}{(N-1)^{2}}\left[\ln \left(\frac{s_{N}}{s_{1}}\right)\right]^{2}
$$

The phase only condition used to derive equations (1.5) and (1.6) is equivalent to modelling the stochastic function $u(t)$ in terms of a random walk in the (complex) Fourier domain where the amplitude of each step is the same.

\section{Derivation of the Generalised Kolmogorov-Feller Equation}

For an arbitrary Characteristic Function $P(k)$ with Probability Density Function (PDF) $p(x)$, Einstein's evolution equation is, Einstein, 1905

$$
u(x, t+\tau)=u(x, t) \otimes_{x} p(x)
$$


where $u(x, t)$ is a 'density function' representing the concentration of a canonical ensemble of particles undergoing elastic collisions. Consider a Taylor series for the function $u(x, t+\tau)$, i.e.

$$
u(x, t+\tau)=u(x, t)+\tau \frac{\partial}{\partial t} u(x, t)+\frac{\tau^{2}}{2 !} \frac{\partial^{2}}{\partial t^{2}} u(x, t)+\ldots
$$

For $\tau<<1$

$$
u(x, t+\tau)=u(x, t)+\tau \frac{\partial}{\partial t} u(x, t)
$$

and we obtain the 'Classical KFE' (Kolmogorov, 1931, Feller, 1957)

$$
\tau \frac{\partial}{\partial t} u(x, t)=-u(x, t)+u(x, t) \otimes_{x} p(x)
$$

Equation (1.7) is based on a critical assumption which is that the time evolution of the field $u(x, t)$ is influenced only by short term events and that longer term (historical) events have no influence on the behaviour of the field, i.e. the 'system' described by equation (1.7) has no 'memory'. This statement is the physical basis upon which we introduce the condition $\tau<<1$ thereby allowing the Taylor series expansion of the $u(x, t+\tau)$ to be made to first order. The question then arises as to how longer term temporal influences can be modelled, other than by taking an increasingly larger number of terms in the Taylor expansion of $u(x, t+t a u)$ which is not of practical analytical value. For arbitrary values of $\tau$,

$$
\tau \frac{\partial}{\partial t} u(x, t)+\frac{\tau^{2}}{2 !} \frac{\partial^{2}}{\partial t^{2}} u(x, t)+\ldots=-u(x, t)+u(x, t) \otimes_{x} p(x)
$$

We can model the effect on a solution for $u(x, t)$ of the series on the left hand side of this equation in terms of a 'memory function' $m(t)$ and write

$$
\tau m(t) \otimes_{t} \frac{\partial}{\partial t} u(x, t)=-u(x, t)+u(x, t) \otimes_{x} p(x)
$$

where $\otimes_{t}$ is taken to denote the causal convolution integral over $t$. This is the Generalised KFE (GKFE) which reduces to the Classical KFE when

$$
m(t)=\delta(t)
$$

Note that for any memory function for which there exists a function or class of functions of the type $n(t)$, say, such that

$$
n(t) \otimes_{t} m(t)=\delta(t)
$$


then we can write equation (1.8) in the form

$$
\tau \frac{\partial}{\partial t} u(x, t)=-n(t) \otimes_{t} u(x, t)+n(t) \otimes_{t} u(x, t) \otimes_{x} p(x)
$$

where the Classical KFE is recovered when $n(t)=\delta(t)$.

Any solution obtained to the GKFE will be dependent upon the choice of memory function $m(t)$ used. There are a number of choices that can be considered, each or which is taken to be a 'best characteristic' of the stochastic system in terms of the influence of its time history. However, it may be expected that the time history of physically significant random systems is relatively localised in time. This includes memory functions such as the Mittag-Leffler function, Olver and Maximon, 2010

$$
m(t)=\frac{1}{\Gamma(1-\beta) t^{\beta}}, \quad 0<\beta<1
$$

where

$$
n(t)=\frac{1}{\Gamma(\beta-1) t^{2-\beta}}
$$

given that

$$
\int_{0}^{\infty} \frac{\exp (-s t)}{\Gamma(\beta) t^{1-\beta}} d t=\frac{1}{s^{\beta}} \text { and } \int_{0}^{\infty} \delta(t) \exp (-s t) d t=1
$$

\section{Solution to the GKFE using the Green's Function Method}

Consider equation (1.9) which can be written in the form

$$
\begin{gathered}
\tau \frac{\partial}{\partial t} u(x, t)+u(x, t)=u(x, t)-n(t) \otimes_{t} u(x, t) \\
+n(t) \otimes_{t} u(x, t) \otimes_{x} p(x)
\end{gathered}
$$

so that the Green's function solution is given by

$$
\begin{aligned}
u(x, t)= & g(t) \otimes_{t} u(x, t)-g(t) \otimes_{t} n(t) \otimes_{t} u(x, t) \\
& +g(t) \otimes_{t} n(t) \otimes_{t} u(x, t) \otimes_{x} p(x)
\end{aligned}
$$

where the Green's function is given by

$$
g(t)=\frac{1}{\tau} \exp (-t / \tau), \quad t>0
$$

which is the solution to

$$
\tau \frac{\partial}{\partial t} g\left(t-t_{0}\right)+g\left(t-t_{0}\right)=\delta\left(t-t_{0}\right)
$$


and we assume the initial conditions $u(x, t=0)=0$ and $g\left(t=t_{0}\right)=0$. We can now analyse this solution in Fourier-Laplace space by taking the Fourier transform and the Laplace transform of equation (1.10) and using the convolution theorems for the Fourier and Laplace transform, respectively, to obtain

$$
\overline{\widetilde{u}}(k, s)=\bar{g}(s) \overline{\widetilde{u}}(k, s)-\bar{g}(s) \bar{n}(s) \overline{\widetilde{u}}(k, s)+\bar{g}(s) \bar{n}(s) \overline{\widetilde{u}}(k, s) \widetilde{p}(k)
$$

where

$$
\begin{gathered}
\overline{\widetilde{u}}(k, s)=\int_{0}^{\infty} \int_{-\infty}^{\infty} u(x, t) \exp (-i k x) d x \exp (-s t) d t \\
\bar{g}(s)=\int_{0}^{\infty} g(t) \exp (-s t) d t, \quad \bar{n}(s)=\int_{0}^{\infty} n(t) \exp (-s t) d t
\end{gathered}
$$

and

$$
\widetilde{p}(k)=\int_{-\infty}^{\infty} p(x) \exp (-i k x) d x
$$

From equation (1.11) it is clear that we can write

$$
\begin{gathered}
\overline{\widetilde{u}}(k, s)=-\frac{\bar{g}(s)}{1-\bar{g}(s)} \bar{n}(s) \overline{\widetilde{u}}(k, s)+\frac{\bar{g}(s)}{1-\bar{g}(s)} \bar{n}(s) \overline{\widetilde{u}}(k, s) \widetilde{p}(k) \\
=-\frac{\bar{n}(s)}{\tau s} \overline{\widetilde{u}}(x, t)+\frac{\bar{n}(s)}{\tau s} \overline{\widetilde{u}}(k, s) \widetilde{p}(k)
\end{gathered}
$$

given that $\bar{g}(s)=(1+\tau s)^{-1}$ and thus we obtain the equation

$$
\overline{\widetilde{u}}(k, s)=\bar{h}(s) \overline{\widetilde{u}}(k, s) \widetilde{p}(k)
$$

where

$$
\bar{h}(s)=\frac{\bar{n}(s)}{\tau s+\bar{n}(s)}
$$

or, upon inverse transformations

$$
u(x, t)=h(t) \otimes_{t} u(x, t) \otimes_{x} p(x)
$$

with

$$
h(t) \leftrightarrow \frac{\bar{n}(s)}{\tau s+\bar{n}(s)}
$$

where $\leftrightarrow$ denotes the Laplce transformation, i.e. mutual transformation from $t$-space to $s$-space. 
Consider the iteration of equation (1.13) defined by

$$
u_{n+1}(x, t)=h(t) \otimes_{t} u_{n}(x, t) \otimes_{x} p(x)
$$

for an initial solution $u_{0}(x, t)$ where $n=1,2, \ldots, N$ The equivalent iteration in Fourier-Laplace space is, from equation (1.12)

$$
\overline{\widetilde{u}}_{n+1}(k, s)=\bar{h}(s) \overline{\widetilde{u}}_{n}(k, s) \widetilde{p}(k)
$$

with initial solution $\overline{\widetilde{u}}_{0}(k, s)$. From equation (15) it is clear that, after $N$ iterations, we can write

$$
\overline{\widetilde{u}}_{N}(k, s)=[\bar{h}(s)]^{N}[\widetilde{p}(k)]^{N} \overline{\widetilde{u}}_{0}(k, s)
$$

so that upon inverse Fourier-Laplace transformation, equation (1.14) becomes

$$
u_{N}(x, t)=\prod_{j=1}^{N} p(x) \prod_{k=1}^{N} h(t) \otimes_{x} \otimes_{t} u_{0}(x, t)
$$

where

$$
\prod_{j=1}^{N} f(t) \equiv f(t) \otimes_{t} f(t) \otimes_{t} f(t) \otimes_{t} \ldots
$$

denoting the $N^{\text {th }}$ convolution of $f(t)$. The convergence criterion required for the iteration defined by equation (1.14) is given in the Appendix A.

\section{Mittag-Leffler Impulse Response Function}

Form equation (1.16), if the initial solution is an impulse (i.e. $u_{0}(x, t)=$ $\delta(x) \delta(t)$ then the Impulse Response Function (IRF), denoted by $r(x, t)$, is given by

$$
r(x, t)=\prod_{j=1}^{N} p(x) \prod_{k=1}^{N} h(t)
$$

with 'transfer function'

$$
\overline{\widetilde{r}}(k, s)=[\bar{h}(s) \widetilde{p}(k)]^{N}
$$

For a memory function $m(t)$ modelled by the Mittag-Leffler function (for $0<\beta<1)$

$$
m(t) \leftrightarrow \frac{1}{s^{1-\beta}}, \quad \bar{h}(s)=\frac{1}{1+\tau s^{\beta}} \sim \frac{1}{\tau s^{\beta}}
$$

so that

$$
h(t) \sim \frac{1}{\tau \Gamma(\beta) t^{1-\beta}}
$$


Similarly, suppose we consider a Mittag-Leffler PDF of the form

$$
p(x)=\frac{1}{\Gamma(1-\gamma)|x|^{\gamma}}, \quad 0<\gamma<1
$$

so that the IRF becomes

$$
r(x, t) \sim \prod_{j=1}^{N} \frac{1}{\Gamma(1-\gamma)|x|^{\gamma}} \prod_{k=1}^{N} \frac{1}{\tau \Gamma(\beta) t^{1-\beta}}
$$

Note that, from Appendix A, if $\|h(t)\| \times\|p(x)\|<<1$ then $r(x, t) \sim$ $p(x) h(t)$, and, in the case of the Mittag-Leffler function used here, this will occur when $\tau>>1$. Also, note that $r(x, t) \rightarrow 0$ as $\gamma \rightarrow 1$ and as $\beta \rightarrow 0$.

\section{Trend Analysis using MetaTrader4}

On the basis of the results discussed in the previous section, we consider a short time series model given by (for an arbitrary PDF $p$ )

$$
\hat{u}(t) \equiv \int_{-\infty}^{\infty} p(x) h(t) d x=\frac{a}{t^{1-\beta}}, \quad \beta>0
$$

where $a$ is a scaling constant. This model represents the IRF associated with a random scaling fractal signal $u(t)$, Blackledge, 2010. For the discrete case when $\hat{u}_{n} \equiv \hat{u}\left(t_{n}\right)$ (for $\left.n=1,2, \ldots, N\right)$ is taken to represent a window of data taken from an input data steam,

$$
\hat{u}_{n}=a t_{n}^{\alpha}, \quad t_{n}>0
$$

where $\alpha=\beta-1$. Estimates of the parameters $a$ and $\alpha$ are then chosen to minimise the error function

$$
e(a, \alpha)=\left\|\ln \hat{u}_{n}-\ln u_{n}\right\|_{2}^{2} \equiv \sum_{n=1}^{N}\left(\ln \hat{u}_{n}-\ln u_{n}\right)^{2}
$$

where $u_{n}$ is data which is taken to be normalised, i.e. $\left\|u_{n}\right\|_{\infty}=1$. Differentiating with respect to $A=\ln a$ and $\alpha$, it is trivial to show that

$$
\alpha=\frac{\sum_{n=1}^{N} \ln u_{n} \sum_{n=1}^{N} \ln t_{n}-N \sum_{n=1}^{N} \ln u_{n} \ln t_{n}}{\left(\sum_{n=1}^{N} \ln t_{n}\right)^{2}-N \sum_{n=1}^{N}\left(\ln t_{n}\right)^{2}}
$$


and

$$
a=\exp \left(\frac{\sum_{n=1}^{N} \ln u_{n}-\alpha \sum_{n=1}^{N} \ln t_{n}}{N}\right)
$$

given that

$$
\frac{\partial e}{\partial \alpha}=0 \text { and } \frac{\partial e}{\partial A}=0
$$

Note that in general, $\alpha=\beta-1$ ) may be greater than (for $\beta>1$ ) or less than (for $0<\beta<1$ ) zero thereby providing a measure of any (long term) ascending or descending trends in the data $u_{n}$, respectively. An example of this characteristic, coupled with the corresponding Stochastic Volatility of the same financial times series is given in Figure 1.1, the version of Metatrader4 used for this application being available from Alpari Limited, Alpari, 2012. This figure shows the results of computing the Stochastic Volatility using equation (1.6) and the $\alpha$-index using equation (1.17) for daily sampled 'Gold Spot' data (XAGUSD, Daily) from 29 August to 20 September, 2012. This figure shows a window of data over which there are both downward and upward trends of roughly equal range and rate. Note that the Stochastic Volatility decays over the latter upward trend when the XAUUSD, Daily increases from approximately 1573 (on 25 July, 2012) to 1772 (on 1 October, 2012) USD/oz. This result is typical of observations indicating that an increase in the value of the $\alpha$-index coupled with a decrease in the volatility provides an appropriate 'investment signature'.

A further example is given in Figure 1.2 which shows the $\alpha$-index, and, as a comparator, the Lyapunov exponent $\lambda$ computed via the equation

$$
\lambda=\frac{1}{N} \sum_{i=1}^{N} \log \left|\frac{u_{n+1}}{u_{n}}\right|
$$

for EUA (European Union Allowance) Carbon Dioxide Emissions using hourly sampled data (EUA, H1) from 17 August to 5 October, 2012. It is noted that the Lyaponuv exponent exhibits similar behaviour to the $\alpha$-index but that the latter index is smoother (for the same period used).

\section{Conclusion}

Compared to equations such as the Classical Diffusion and Fractional Diffusion Equations, Gorenflo et al., 2000, the GKFE given by equation (1.8) represents a more accurate model for a density function describing random motion that conforms to Einstein's evolution equation. We have 


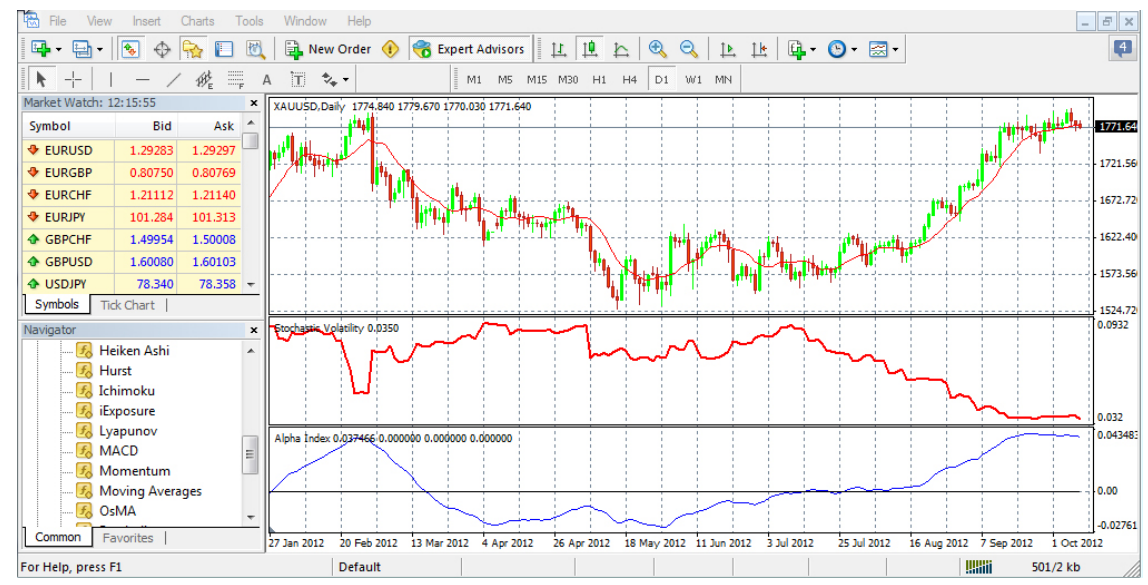

Figure 1.1. The Gold Spot Price (XAUUSD, Daily) from 27 January to 1 October, 2012 (top window), the Stochastic Volatility (centre window) computed using equation (1.6) for a period of 50 days and the $\alpha$-index (lower window) computed using equation (1.17) also for a period of 50 days.

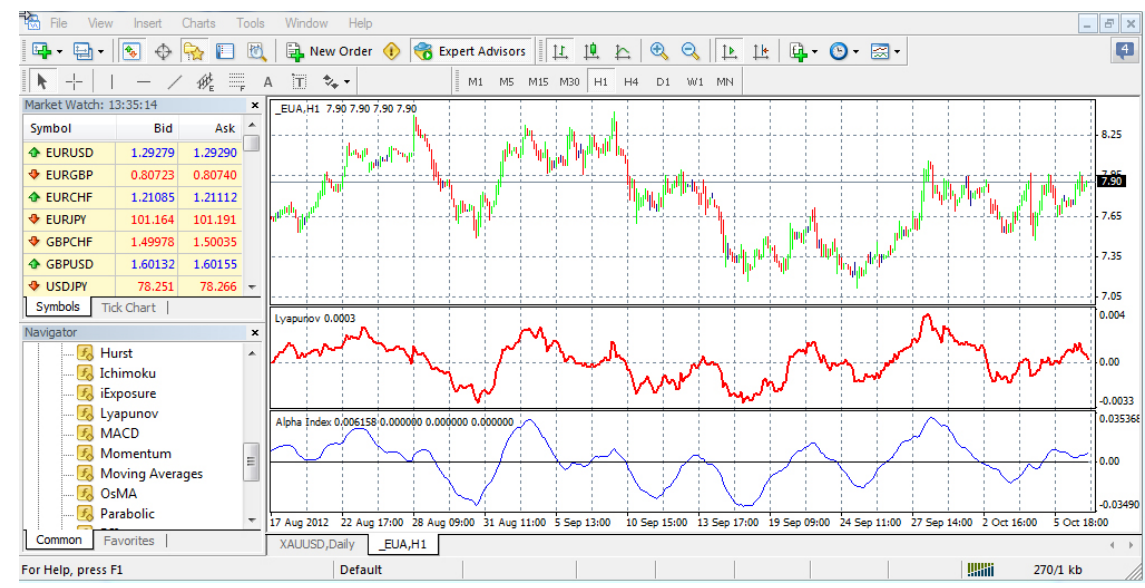

Figure 1.2. European Union Allowance $\mathrm{CO}_{2}$ emissions (EUA, H1) from 17 August to 5 October, 2012 (top window), the Lyapunov exponent (centre window) computed using equation (1.18) for a period of 30 and the $\alpha$-index (lower window) using equation (1.17) also computed for a period of 30.

considered the Green's function solution of the GKFE as a model for a financial time series (or a derived index). The time dependence of this solution depends upon the memory function used to model the higher order terms in the Taylor series expansion of the evolution equation, and, in this paper, we have used the Mittag-Leffler memory function. It has 
been shown that this choice provides a temporal solution that scales at $t^{\alpha}$ where $\alpha=\beta-1,0<\beta<1$. For $\beta>0$ the parameter $\alpha$ provides an index that identifies the start, direction and end of a trend depending on the position in time where the polarity of $\alpha$ changes from being positive (indicating an upward trend) to negative (indicating a downward trend). Coupled with knowledge of the Stochastic Volatility being relative low, this index therefore provides a quantitative measure for implementing a trading strategy that is predicating on forecasting the type and extent of a trend. For readers interested in further evaluating this approach to trend analysis, the MetaTrader 4 .mq4 modules used to obtain the results given in the previous section are available from MT4 Indicators URL, 2012.

\section{APPENDIX 1.A: Condition for Convergence of Equa- tion (1.14)}

Consider the error function $\epsilon_{n}(x, t)$ at any iteration $n$ so that $u_{n}(x, t)=u(x, t)+$ $\epsilon_{n}(x, t)$ From equation (1.15) we can then write

$$
\overline{\widetilde{\epsilon}}_{n+1}(k, s)=\bar{h}(s) \widetilde{p}(k) \overline{\widetilde{\epsilon}}_{n}(k, s)
$$

so that

$$
\overline{\widetilde{\epsilon}}_{n}(k, s)=[\bar{h}(s) \widetilde{p}(k)]^{n} \overline{\widetilde{\epsilon}}_{0}(k, s)
$$

and it is clear that, since we require $\overline{\widetilde{\epsilon}}_{n} \rightarrow 0$ and $n \rightarrow \infty,[\bar{h}(s) \widetilde{p}(k)]<1 \quad \forall(k, s)$. The condition for convergence therefore becomes

$$
\|\bar{h}(s) \widetilde{p}(k)\| \leq\|\bar{h}(s)\| \times\|\widetilde{p}(k)\|<1
$$

or, for Euclidian norms, and, using Rayleigh's theorem,

$$
\|\bar{h}(s)\|_{2} \times\|p(x)\|_{2}<\frac{1}{\sqrt{2 \pi}}
$$

In $(k, t)$-space

$$
\widetilde{\epsilon}_{n}(k, t)=\prod_{k=1}^{n} h(t)[\widetilde{p}(k)]^{n} \otimes_{t} \widetilde{\epsilon}_{0}(k, t)
$$

so that, using Hölder's inequaility

$$
\begin{gathered}
\left\|\widetilde{\epsilon}_{n}(k, t)\right\| \leq\left\|\prod_{k=1}^{n} h(t)[\widetilde{p}(k)]^{n}\right\| \times\left\|\widetilde{\epsilon}_{0}(k, t)\right\| \\
\leq\|h(t)\|^{n} \times\|\widetilde{p}(k)\|^{n} \times\left\|\widetilde{\epsilon}_{0}(k, t)\right\|
\end{gathered}
$$

and the condition for convergence becomes

$$
\|h(t)\|_{2} \times\|p(x)\|_{2}<\frac{1}{\sqrt{2 \pi}}
$$

\section{Acknowledgment}

The authors acknowledges the support of the Science Foundation Ireland and Enterprise Ireland. 


\section{References}

Blackledge, J. M., Lamphiere, M., Murphy, K., Overton, S. and Panahi, A. (2012) "Stochastic Volatility Analysis using the Generalised Kolmogorov-Feller Equation", Lecture Notes in Engineering and Computer Science: Proceedings of The World Congress on Engineering 2012, WCE 2012, 4-6 July, 2012, London, U.K., 453-458

Blackledge, J. M. (2010). The Fractal Market Hypothesis: Applications to Financial Forecasting, Centre for Advanced Studies, Warsaw University of Technology, Poland, ISBN: 978-83-61993-01-83.

Lamoureux, C. G. and Lastrapes, W. D. (1993). "Forecasting Stock-Return Variance: Toward an Understanding of Stochastic Implied Volatilities", Review of Financial Studies, 6:293-326.

Wiggins, J. B. (1987). "Option Values under Stochastic Volatilities", Journal of Financial Economics, 19:351-372, 1987.

Melino A. and Turnbull S. (1990). "The Pricing of Foreign Currency Options with Stochastic Volatility;, Journal of Econometrics, 45: 239-265.

Stein, E. M. and Stein, J. C. (1991). "Stock Price Distributions with Stochastic Volatility: An Analytic Approach", Review of Financial Studies, 4: 727-752.

Einstein, A. (1905). "On the Motion of Small Particles Suspended in Liquids at Rest Required by the Molecular-Kinetic Theory of Heat", Annalen der Physik, 17: 549560

Kolmogorov, A. N. (1992), "On Analytic Methods in Probability Theory", Selected Works of A. N. Kolmogorov, Volume II: Probability Theory and Mathematical Statistics (Ed. A. N. Shiryaev), Kluwer, Dordrecht, 61-108. (From the Original: Uber die Analytischen Methoden in der Wahrscheinlichkeitsrechnung, (1931). Math. Ann. 104: 415-458).

Feller W. (1957). "On Boundaries and Lateral Conditions for the Kolmogorov Differential Equations", The Annals of Mathematics, Second Series, 65(3): 527-570.

Olver, F. W. and Maximon, L. C. (2010). Mittag-Leffler function, Handbook of Mathematical Functions in Olver, (Eds. W. J. Frank et al.), NIST, Cambridge University Press.

Alpari, 2012 Limited is a broker headquartered in London for Forex, Spread Betting, Precious Metals, Futures and Energy Commodities markets and provides a range of trading platforms including MetaTrader 4.

http://www.alpari.co.uk/

Gorenflo, R., Mainardi, F., Raberto, M. and Scalas, E. (2000), "Fractional Diffusion in Finance: Basic Theory, A Review Paper Based on a Talk Given by F. Mainardi at MDEF2000 - Workshop 'Modelli Dinamici in Economia e Finanza', Urbino (Italy), September 28-30, 2000.

http://www. econ.uniurb.it/bischi/MDEF2000/MainardiMDEF.pdf

MT4 Indicators URL, 2012. The MetaTrader4 indicators used to generates the results given in this paper are available from http://eleceng.dit.ie/jblackledge/ Indicators .zip which provides the .mq4 modules requires to compute the Stochastic Volatility, the $\alpha$-index and the Lyapunov exponent. 